PROCEEDINGS OF THE

AMERICAN MATHEMATICAL SOCIETY

Volume 140, Number 3, March 2012, Pages 795-804

S 0002-9939(2011)10957-2

Article electronically published on July 20, 2011

\title{
THE NUMBER OF GOLDBACH REPRESENTATIONS OF AN INTEGER
}

\author{
ALESSANDRO LANGUASCO AND ALESSANDRO ZACCAGNINI
}

(Communicated by Matthew A. Papanikolas)

ABstract. Let $\Lambda$ be the von Mangoldt function and $R(n)=\sum_{h+k=n} \Lambda(h) \Lambda(k)$
be the counting function for the Goldbach numbers. Let $N \geq 2$ and assume that the Riemann Hypothesis holds. We prove that

$$
\sum_{n=1}^{N} R(n)=\frac{N^{2}}{2}-2 \sum_{\rho} \frac{N^{\rho+1}}{\rho(\rho+1)}+\mathcal{O}\left(N \log ^{3} N\right),
$$

where $\rho=1 / 2+i \gamma$ runs over the non-trivial zeros of the Riemann zeta-function $\zeta(s)$. This improves a recent result by Bhowmik and Schlage-Puchta.

\section{INTRODUCTION}

Let $\Lambda$ be the von Mangoldt function and

$$
R(n)=\sum_{h_{1}+h_{2}=n} \Lambda\left(h_{1}\right) \Lambda\left(h_{2}\right)
$$

be the counting function for the Goldbach numbers. This paper is devoted to studying the behaviour of the average order of magnitude of $R(n)$ for $n \in[1, N]$, where $N$ is a large integer. We have the following

Theorem 1.1. Let $N \geq 2$ and assume the Riemann Hypothesis (RH) holds. Then

$$
\sum_{n=1}^{N} R(n)=\frac{N^{2}}{2}-2 \sum_{\rho} \frac{N^{\rho+1}}{\rho(\rho+1)}+\mathcal{O}\left(N \log ^{3} N\right),
$$

where $\rho=1 / 2+i \gamma$ runs over the non-trivial zeros of the Riemann zeta function $\zeta(s)$.

The first result of this kind was proved in 1991 by Fujii, who subsequently improved it (see [4]-6]) until reaching the error term $\mathcal{O}\left((N \log N)^{4 / 3}\right)$. Then Granville [8]-9] gave an alternative proof of the same result and, finally, Bhowmik and Schlage-Puchta [2] were able to reach the error term $\mathcal{O}\left(N \log ^{5} N\right)$. In [2] they also proved that the error term is $\Omega(N \log \log N)$.

Our result improves the upper bound in Bhowmik and Schlage-Puchta 2 by a factor of $\log ^{2} N$. In fact, this seems to be the limit of the method in the current state of the circle-method technology; see the remark after the proof.

Received by the editors November 11, 2010 and, in revised form, December 16, 2010.

2010 Mathematics Subject Classification. Primary 11P32; Secondary 11P55.

Key words and phrases. Goldbach-type theorems, Hardy-Littlewood method.

(C)2011 American Mathematical Society Reverts to public domain 28 years from publication 
If one admits the presence of some suitable weight in our average, a sharper result can be obtained. For example, using the Fejér weight we could work with $L(N ; \alpha)=\sum_{n=-N}^{N}(N-|n|) e(n \alpha)=|T(N ; \alpha)|^{2}$ instead of $T(N ; \alpha)$ in (4.2). The key property is that, for $1 / N<|\alpha| \leq 1 / 2$, the function $L(N ; \alpha)$ decays as $\alpha^{-2}$ instead of $|\alpha|^{-1}$, and so the dissection argument in (4.5) is now more efficient and does not cause any loss of logs. Such a phenomenon is well-known from the literature about the existence of Goldbach numbers in short intervals; see, e.g., Languasco and Perelli [12].

In fact we will obtain Theorem 1.1 as a consequence of a weighted result. Letting $\psi(x)=\sum_{m \leq x} \Lambda(m)$, we have

Theorem 1.2. Let $2 \leq y \leq N$ and assume the Riemann Hypothesis (RH) holds. Then

$$
\max _{y \in[2, N]}\left|\sum_{n=1}^{y}[R(n)-(2 \psi(n)-n)] e^{-n / N}\right| \ll N \log ^{3} N .
$$

The key reason why we are able to derive Theorem 1.1 from Theorem 1.2 via partial summation is that the exponential weight in (1.1) just varies in the range $\left[e^{-1 / N}, e^{-1}\right]$, and so it does not change the order of magnitude of the functions involved.

We will use the original Hardy and Littlewood [10] circle method setting, i.e., the weighted exponential sum

$$
\widetilde{S}(\alpha)=\sum_{n=1}^{\infty} \Lambda(n) e^{-n / N} e(n \alpha),
$$

where $e(x)=\exp (2 \pi i x)$, since it lets us avoid the use of Gallagher's Lemma (Lemma 1 of [7]) and hence, in this conditional case, it gives slightly sharper results; see Lemma 2.1 below. Such a function was also used by Linnik [13, 14. The new ingredient in this paper is Lemma 2.5 below in which we unconditionally detect the existence of the term $-2 \sum_{\rho} N^{\rho+1} /(\rho(\rho+1))$ connecting it to a suitable average of the difference $\psi(n)-n$. In the previously mentioned papers this is obtained by applying the explicit formula for $\psi(n)$ twice.

The ideas that led to Theorems 1.1 and 1.2 also work for the sum of $k \geq 3$ primes, i.e., for the function

$$
R_{k}(n)=\sum_{h_{1}+\ldots+h_{k}=n} \Lambda\left(h_{1}\right) \cdots \Lambda\left(h_{k}\right)
$$

We can prove the following.

Theorem 1.3. Let $k \geq 3$ be an integer, $N \geq 2$ and assume the Riemann Hypothesis (RH) holds. Then

$$
\sum_{n=1}^{N} R_{k}(n)=\frac{N^{k}}{k !}-k \sum_{\rho} \frac{N^{\rho+k-1}}{\rho(\rho+1) \cdots(\rho+k-1)}+\mathcal{O}_{k}\left(N^{k-1} \log ^{k} N\right),
$$

where $\rho=1 / 2+i \gamma$ runs over the non-trivial zeros of $\zeta(s)$.

The proof of Theorem 1.3 is completely similar to the one of Theorems 1.1 and 1.2. We just remark that the main differences are in the use of the explicit formula 
for

$$
\psi_{j}(t):=\frac{1}{j !} \sum_{n \leq t}(t-n)^{j} \Lambda(n),
$$

where $j$ is a non-negative integer, and of the following version of Lemma 1 of [1]:

Lemma. Assume the Riemann Hypothesis (RH) holds. Let $N \geq 2, z=1 / N-2 \pi i \alpha$ and $\alpha \in[-1 / 2,1 / 2]$. Then

$$
\left|\widetilde{S}(\alpha)-\frac{1}{z}\right| \ll N^{1 / 2}\left(1+(N|\alpha|)^{1 / 2}\right) \log N
$$

Another connected problem we can address with this technique is a short-interval version of Theorem 1.1. We can prove the following.

Theorem 1.4. Let $2 \leq H \leq N$ and assume the Riemann Hypothesis (RH) holds. Then

$$
\sum_{n=N}^{N+H} R(n)=H N+\frac{H^{2}}{2}-2 \sum_{\rho} \frac{(N+H)^{\rho+1}-N^{\rho+1}}{\rho(\rho+1)}+\mathcal{O}\left(N \log ^{2} N \log H\right),
$$

where $\rho=1 / 2+i \gamma$ runs over the non-trivial zeros of $\zeta(s)$.

Also in this case we do not give a proof of Theorem 1.4. We just remark that the main difference is in the use of the exponential sum $\sum_{n=N}^{N+H} e(n \alpha)$ instead of $\sum_{n=1}^{N} e(n \alpha)$.

\section{Setting of the CirCle Method}

For brevity, throughout the paper we write

$$
z=\frac{1}{N}-2 \pi i \alpha, \quad \text { where } N \text { is a large integer and } \alpha \in[-1 / 2,1 / 2] .
$$

The first lemma is an $L^{2}$-estimate for the difference $\widetilde{S}(\alpha)-1 / z$.

Lemma 2.1 (Languasco and Perelli [12). Assume RH. Let $N$ be a sufficiently large integer and $z$ be as in (2.1). For $0 \leq \xi \leq 1 / 2$, we have

$$
\int_{-\xi}^{\xi}\left|\widetilde{S}(\alpha)-\frac{1}{z}\right|^{2} \mathrm{~d} \alpha \ll N \xi \log ^{2} N
$$

This follows immediately from the proof of Theorem 1 of [12] since the quantity we would like to estimate here is $\widetilde{R}_{1}+\widetilde{R}_{3}+\widetilde{R}_{5}$ there.

Lemma 2.1 is the main reason why we use $\widetilde{S}(\alpha)$ instead of its truncated form $S(\alpha)=\sum_{n=1}^{N} \Lambda(n) e(n \alpha)$ as in Bhowmik and Schlage-Puchta [2]. In fact Lemma2.1 lets us avoid the use of Gallagher's Lemma 7], which leads to a loss of a factor $\log ^{2} N$ in the final estimate (compare Lemma 2.1 with Lemma 4 of [2]). For a similar phenomenon in a slightly different situation, see also Languasco [11.

The next four lemmas do not depend on $\mathrm{RH}$. By the residue theorem one can obtain

Lemma 2.2 (Eq. (29) of [12]). Let $N \geq 2,1 \leq n \leq N$ and $z$ be as in (2.1). We have

$$
\int_{-\frac{1}{2}}^{\frac{1}{2}} \frac{e(-n \alpha)}{z^{2}} \mathrm{~d} \alpha=n e^{-n / N}+\mathcal{O}(1)
$$

uniformly for every $n \leq N$. 
Lemma 2.3. Let $N$ be a sufficiently large integer and $z$ be as in (2.1). We have

$$
\int_{-\frac{1}{2}}^{\frac{1}{2}}\left|\widetilde{S}(\alpha)-\frac{1}{z}\right|^{2} \mathrm{~d} \alpha=\frac{N}{2} \log N+\mathcal{O}\left(N(\log N)^{1 / 2}\right) .
$$

Proof. By the Parseval theorem and the Prime Number Theorem we have

$$
\int_{-\frac{1}{2}}^{\frac{1}{2}}|\widetilde{S}(\alpha)|^{2} \mathrm{~d} \alpha=\sum_{m=1}^{\infty} \Lambda^{2}(m) e^{-2 m / N}=\frac{N}{2} \log N+\mathcal{O}(N) .
$$

Recalling that the equation at the beginning of page 318 of $[12$ implies

$$
\int_{-\frac{1}{2}}^{\frac{1}{2}} \frac{\mathrm{d} \alpha}{|z|^{2}}=\frac{N}{\pi} \arctan (\pi N)
$$

the lemma immediately follows using the relation $|a-b|^{2}=|a|^{2}+|b|^{2}-2 \Re(a \bar{b})$ and the Cauchy-Schwarz inequality.

Let

$$
V(\alpha)=\sum_{m=1}^{\infty} e^{-m / N} e(m \alpha)=\sum_{m=1}^{\infty} e^{-m z}=\frac{1}{e^{z}-1} .
$$

Lemma 2.4. If $z$ satisfies (2.1), then $V(\alpha)=z^{-1}+\mathcal{O}(1)$.

Proof. We recall that the function $w /\left(e^{w}-1\right)$ has a power-series expansion with radius of convergence $2 \pi$ (see for example Apostol [1, page 264). In particular, uniformly for $|w| \leq 4<2 \pi$ we have $w /\left(e^{w}-1\right)=1+\mathcal{O}(|w|)$. Since $z$ satisfies (2.1) we have $|z| \leq 4$, and the result follows.

Now let

$$
T(y ; \alpha)=\sum_{n=1}^{y} e(n \alpha) \ll \min \left(y ; \frac{1}{\|\alpha\|}\right) .
$$

Lemma 2.5. Let $N$ be a large integer, $2 \leq y \leq N$ and $z$ be as in (2.1). We have

$$
\int_{-1 / 2}^{1 / 2} T(y ;-\alpha) \frac{(\widetilde{S}(\alpha)-1 / z)}{z} \mathrm{~d} \alpha=\sum_{n=1}^{y} e^{-n / N}(\psi(n)-n)+\mathcal{O}\left((y N \log N)^{1 / 2}\right) .
$$

We remark that Lemma 2.5 is unconditional, and hence it implies, also using Lemma 2.6, that the ability of detecting the term depending on the zeros of the Riemann $\zeta$-function in Theorem 1.1 does not depend on RH.

Proof. Writing $\widetilde{R}(\alpha)=\widetilde{S}(\alpha)-1 / z$, by Lemma 2.4 we have

$$
\begin{aligned}
\int_{-1 / 2}^{1 / 2} T(y ;-\alpha) \frac{\widetilde{R}(\alpha)}{z} \mathrm{~d} \alpha & =\int_{-1 / 2}^{1 / 2} T(y ;-\alpha) \widetilde{R}(\alpha) V(\alpha) \mathrm{d} \alpha \\
& +\mathcal{O}\left(\int_{-1 / 2}^{1 / 2}|T(y ;-\alpha)||\widetilde{R}(\alpha)| \mathrm{d} \alpha\right) \\
& =\int_{-1 / 2}^{1 / 2} T(y ;-\alpha) \widetilde{R}(\alpha) V(\alpha) \mathrm{d} \alpha+\mathcal{O}\left((y N \log N)^{1 / 2}\right),
\end{aligned}
$$


since, by the Parseval theorem and Lemma 2.3, the error term above is

$$
\ll\left(\int_{-1 / 2}^{1 / 2}|T(y ;-\alpha)|^{2} \mathrm{~d} \alpha\right)^{1 / 2}\left(\int_{-1 / 2}^{1 / 2}|\widetilde{R}(\alpha)|^{2} \mathrm{~d} \alpha\right)^{1 / 2} \ll(y N \log N)^{1 / 2} .
$$

Again by Lemma 2.4 we have

$$
\widetilde{R}(\alpha)=\widetilde{S}(\alpha)-\frac{1}{z}=\widetilde{S}(\alpha)-V(\alpha)+\mathcal{O}(1)
$$

and hence (2.5) implies

$$
\begin{aligned}
\int_{-1 / 2}^{1 / 2} T(y ;-\alpha) \frac{\widetilde{R}(\alpha)}{z} \mathrm{~d} \alpha & =\int_{-1 / 2}^{1 / 2} T(y ;-\alpha)(\widetilde{S}(\alpha)-V(\alpha)) V(\alpha) \mathrm{d} \alpha \\
& +\mathcal{O}\left(\int_{-1 / 2}^{1 / 2}|T(y ;-\alpha)||V(\alpha)| \mathrm{d} \alpha\right)+\mathcal{O}\left((y N \log N)^{1 / 2}\right) .
\end{aligned}
$$

The Cauchy-Schwarz inequality and the Parseval theorem imply that

$$
\begin{aligned}
\int_{-1 / 2}^{1 / 2}|T(y ;-\alpha)||V(\alpha)| \mathrm{d} \alpha & \leq\left(\int_{-1 / 2}^{1 / 2}|T(y ;-\alpha)|^{2} \mathrm{~d} \alpha\right)^{1 / 2}\left(\int_{-1 / 2}^{1 / 2}|V(\alpha)|^{2} \mathrm{~d} \alpha\right)^{1 / 2} \\
& \ll\left(y \sum_{m=1}^{\infty} e^{-2 m / N}\right)^{1 / 2} \ll(y N)^{1 / 2} .
\end{aligned}
$$

By (2.6)-(2.7), we have

$$
\begin{gathered}
\int_{-1 / 2}^{1 / 2} T(y ;-\alpha) \frac{\widetilde{R}(\alpha)}{z} \mathrm{~d} \alpha=\int_{-1 / 2}^{1 / 2} T(y ;-\alpha)(\widetilde{S}(\alpha)-V(\alpha)) V(\alpha) \mathrm{d} \alpha \\
+\mathcal{O}\left((y N \log N)^{1 / 2}\right) .
\end{gathered}
$$

Now, by (1.2) and (2.2) we can write

$$
\widetilde{S}(\alpha)-V(\alpha)=\sum_{m=1}^{\infty}(\Lambda(m)-1) e^{-m / N} e(m \alpha)
$$

so that

$$
\begin{aligned}
& \int_{-1 / 2}^{1 / 2} T(y ;-\alpha)(\widetilde{S}(\alpha)-V(\alpha)) V(\alpha) \mathrm{d} \alpha \\
& =\sum_{n=1}^{y} \sum_{m_{1}=1}^{\infty}\left(\Lambda\left(m_{1}\right)-1\right) e^{-m_{1} / N} \sum_{m_{2}=1}^{\infty} e^{-m_{2} / N} \int_{-1 / 2}^{1 / 2} e\left(\left(m_{1}+m_{2}-n\right) \alpha\right) \mathrm{d} \alpha \\
& =\sum_{n=1}^{y} \sum_{m_{1}=1}^{\infty}\left(\Lambda\left(m_{1}\right)-1\right) e^{-m_{1} / N} \sum_{m_{2}=1}^{\infty} e^{-m_{2} / N} \begin{cases}1 & \text { if } m_{1}+m_{2}=n \\
0 & \text { otherwise }\end{cases} \\
& =\sum_{n=1}^{y} e^{-n / N} \sum_{m_{1}=1}^{n-1}\left(\Lambda\left(m_{1}\right)-1\right)=\sum_{n=1}^{y} e^{-n / N}(\psi(n-1)-(n-1)),
\end{aligned}
$$

since the condition $m_{1}+m_{2}=n$ implies that both variables are $<n$. Now $\psi(n)=$ $\psi(n-1)+\Lambda(n)$ so that

$$
\sum_{n=1}^{y} e^{-n / N}(\psi(n-1)-(n-1))=\sum_{n=1}^{y} e^{-n / N}(\psi(n)-n)+\mathcal{O}(y) .
$$


By (2.8)-(2.9) and the previous equation, we have

$$
\begin{aligned}
\int_{-1 / 2}^{1 / 2} T(y ;-\alpha) \frac{\widetilde{R}(\alpha)}{z} \mathrm{~d} \alpha & =\sum_{n=1}^{y} e^{-n / N}(\psi(n)-n)+\mathcal{O}\left(y+(y N \log N)^{1 / 2}\right) \\
& =\sum_{n=1}^{y} e^{-n / N}(\psi(n)-n)+\mathcal{O}\left((y N \log N)^{1 / 2}\right)
\end{aligned}
$$

since $y \leq N$, and hence (2.4) is proved.

Lemma 2.6. Let $M>1$ be an integer. We have that

$$
\sum_{n=1}^{M}(\psi(n)-n)=-\sum_{\rho} \frac{M^{\rho+1}}{\rho(\rho+1)}+\mathcal{O}(M)
$$

Proof. We recall the definition of $\psi_{0}(t)$ as $\psi(t)-\Lambda(t) / 2$ if $t$ is an integer and as $\psi(t)$ otherwise. Hence

$$
\sum_{n=1}^{M} \psi(n)=\sum_{n=1}^{M} \psi_{0}(n)+\frac{1}{2} \sum_{n=1}^{M} \Lambda(n)=\sum_{n=1}^{M} \psi_{0}(n)+\mathcal{O}(M)
$$

by the Prime Number Theorem. Using the fact that $\psi_{0}(n)=\psi_{0}(t)$ for every $t \in(n, n+1)$, we also get

$$
\sum_{n=1}^{M} \psi_{0}(n)=\sum_{n=1}^{M} \int_{n}^{n+1} \psi_{0}(t) \mathrm{d} t=\int_{0}^{M} \psi_{0}(t) \mathrm{d} t+\mathcal{O}(M)
$$

Remarking that

$$
\sum_{n=1}^{M} n=\int_{0}^{M} t \mathrm{~d} t+\mathcal{O}(M)
$$

we can write

$$
\sum_{n=1}^{M}(\psi(n)-n)=\int_{0}^{M}\left(\psi_{0}(t)-t\right) \mathrm{d} t+\mathcal{O}(M)=\int_{2}^{M}\left(\psi_{0}(t)-t\right) \mathrm{d} t+\mathcal{O}(M)
$$

since the integral on $(0,2]$ gives a contribution of $\mathcal{O}(1)$. For $t \geq 2$ we will use the explicit formula (see eq. (9)-(10) of $\S 17$ of Davenport [3])

$$
\psi_{0}(t)=t-\sum_{|\gamma| \leq Z} \frac{t^{\rho}}{\rho}-\frac{\zeta^{\prime}}{\zeta}(0)-\frac{1}{2} \log \left(1-\frac{1}{t^{2}}\right)+R_{\psi}(t, Z),
$$

where

$$
R_{\psi}(t, Z) \ll \frac{t}{Z} \log ^{2}(t Z)+(\log t) \min \left(1 ; \frac{t}{Z\|t\|}\right) .
$$

The term $-\frac{\zeta^{\prime}}{\zeta}(0)-\frac{1}{2} \log \left(1-\frac{1}{t^{2}}\right)$ gives a contribution of $\mathcal{O}(M)$ to the integral over $[2, M]$ in (2.10). We now need an $L^{1}$ estimate of the error term defined in (2.12). Let

$$
\mathcal{E}(M, Z):=\int_{2}^{M}\left|R_{\psi}(t, Z)\right| \mathrm{d} t .
$$

The first term in (2.12) gives a total contribution to $\mathcal{E}(M, Z)$ which is

$$
\ll \frac{M^{2}}{Z} \log ^{2}(M Z) \text {. }
$$


The second term in (2.12) gives a total contribution to $\mathcal{E}(M, Z)$ which is

$$
\begin{gathered}
\ll \log M \sum_{n=2}^{M}\left[\int_{n}^{n+1 / 2} \min \left(1 ; \frac{t}{Z(t-n)}\right) \mathrm{d} t+\int_{n+1 / 2}^{n+1} \min \left(1 ; \frac{t}{Z(n+1-t)}\right) \mathrm{d} t\right] \\
\ll \log M \sum_{n=2}^{M}\left(\int_{n}^{n+1 / Z} \mathrm{~d} t+\int_{n+1 / Z}^{n+1 / 2} \frac{t \mathrm{~d} t}{Z(t-n)}+\int_{n+1-1 / Z}^{n+1} \mathrm{~d} t\right. \\
\left.+\int_{n+1 / 2}^{n+1-1 / Z} \frac{t \mathrm{~d} t}{Z(n+1-t)}\right) \\
\ll \log M \sum_{n=2}^{M}\left(\frac{3}{Z}+\frac{2 n+1}{Z} \log \left(\frac{Z}{2}\right)\right) \ll \frac{M^{2}}{Z} \log M \log Z .
\end{gathered}
$$

Combining (2.13)-(2.15), for $Z=M \log ^{2} M$ we have that

$$
\mathcal{E}(M, Z) \ll M .
$$

Now inserting (2.11) and (2.16) into (2.10) we obtain

$$
\sum_{n=1}^{M}(\psi(n)-n)=-\sum_{|\gamma| \leq Z} \frac{M^{\rho+1}}{\rho(\rho+1)}+\mathcal{O}(M) .
$$

The lemma follows from (2.17) by remarking that

$$
\sum_{|\gamma|>Z} \frac{M^{\rho+1}}{\rho(\rho+1)} \ll M^{2} \int_{Z}^{+\infty} \frac{\log t}{t^{2}} \mathrm{~d} t \ll \frac{M^{2} \log Z}{Z} \ll \frac{M}{\log M}
$$

since $Z=M \log ^{2} M$.

\section{Proof of Theorem 1.1}

We will obtain Theorem 1.1 as a consequence of Theorem 1.2. By partial summation we have

$$
\begin{aligned}
& \sum_{n=1}^{N}[R(n)-(2 \psi(n)-n)]=\sum_{n=1}^{N} e^{n / N}\left\{[R(n)-(2 \psi(n)-n)] e^{-n / N}\right\} \\
& =e \sum_{n=1}^{N}[R(n)-(2 \psi(n)-n)] e^{-n / N} \\
& \quad-\frac{1}{N} \int_{0}^{N}\left\{\sum_{n=1}^{y}[R(n)-(2 \psi(n)-n)] e^{-n / N}\right\} e^{y / N} \mathrm{~d} y+\mathcal{O}(1) .
\end{aligned}
$$

Inserting (1.1) in (3.1) we get

$$
\sum_{n=1}^{N}[R(n)-(2 \psi(n)-n)] \ll N \log ^{3} N
$$

and hence

$$
\sum_{n=1}^{N} R(n)=\sum_{n=1}^{N} n+2 \sum_{n=1}^{N}(\psi(n)-n)+\mathcal{O}\left(N \log ^{3} N\right) .
$$


Theorem 1.1 now follows by inserting Lemma 2.6 and the identity $\sum_{n=1}^{N} n=N^{2} / 2+$ $\mathcal{O}(N)$ in $(3.2)$.

\section{Proof of Theorem 1.2}

Let $2 \leq y \leq N$. We first recall the definition of the singular series of the Goldbach problem: $\mathfrak{S}(k)=0$ for $k$ odd and

$$
\mathfrak{S}(k)=2 \prod_{p>2}\left(1-\frac{1}{(p-1)^{2}}\right) \prod_{\substack{p \mid k \\ p>2}} \frac{p-1}{p-2}
$$

for $k$ even. Hence, using the well-known estimate $R(n) \ll n \mathfrak{S}(n) \ll n \log \log n$, we remark that

$$
\sum_{n=1}^{y}[R(n)-(2 \psi(n)-n)] e^{-n / N} \ll \sum_{n=1}^{y} n \log \log n \ll y^{2} \log \log y
$$

Therefore, it is clear that (1.1) holds for every $y \in\left[2, N^{1 / 2}\right]$.

Assume now that $y \in\left[N^{1 / 2}, N\right]$ and let $\alpha \in[-1 / 2,1 / 2]$. Writing $\widetilde{R}(\alpha)=$ $\widetilde{S}(\alpha)-1 / z$ and recalling 2.3 we have, say,

$$
\begin{aligned}
& \sum_{n=1}^{y} e^{-n / N} R(n)=\sum_{n=1}^{y} \int_{-\frac{1}{2}}^{\frac{1}{2}} \widetilde{S}(\alpha)^{2} e(-n \alpha) \mathrm{d} \alpha=\int_{-\frac{1}{2}}^{\frac{1}{2}} \widetilde{S}(\alpha)^{2} T(y ;-\alpha) \mathrm{d} \alpha \\
& \quad=\int_{-\frac{1}{2}}^{\frac{1}{2}} \frac{T(y ;-\alpha)}{z^{2}} \mathrm{~d} \alpha+2 \int_{-\frac{1}{2}}^{\frac{1}{2}} \frac{T(y ;-\alpha) \widetilde{R}(\alpha)}{z} \mathrm{~d} \alpha+\int_{-\frac{1}{2}}^{\frac{1}{2}} T(y ;-\alpha) \widetilde{R}(\alpha)^{2} \mathrm{~d} \alpha \\
& (4.2) \quad=I_{1}(y)+I_{2}(y)+I_{3}(y) .
\end{aligned}
$$

Evaluation of $I_{1}(y)$. By Lemma 2.2 we obtain

$$
\begin{aligned}
I_{1}(y) & =\int_{-\frac{1}{2}}^{\frac{1}{2}} \frac{T(y ;-\alpha)}{z^{2}} \mathrm{~d} \alpha=\sum_{n=1}^{y} \int_{-\frac{1}{2}}^{\frac{1}{2}} \frac{e(-n \alpha)}{z^{2}} \mathrm{~d} \alpha=\sum_{n=1}^{y}\left(n e^{-n / N}+\mathcal{O}(1)\right) \\
& =\sum_{n=1}^{y} n e^{-n / N}+\mathcal{O}(y) .
\end{aligned}
$$

Estimation of $I_{2}(y)$. By (2.4) of Lemma 2.5 we obtain

$$
I_{2}(y)=2 \sum_{n=1}^{y} e^{-n / N}(\psi(n)-n)+\mathcal{O}\left((y N \log N)^{1 / 2}\right)
$$


Estimation of $I_{3}(y)$. Using (2.3) and Lemma 2.1] we have that

$$
\begin{aligned}
I_{3}(y) & \ll \int_{-\frac{1}{2}}^{\frac{1}{2}}|T(y ;-\alpha)||\widetilde{R}(\alpha)|^{2} \mathrm{~d} \alpha \\
& \ll y \int_{-\frac{1}{y}}^{\frac{1}{y}}|\widetilde{R}(\alpha)|^{2} \mathrm{~d} \alpha+\int_{\frac{1}{y}}^{\frac{1}{2}} \frac{|\widetilde{R}(\alpha)|^{2}}{\alpha} \mathrm{d} \alpha+\int_{-\frac{1}{2}}^{-\frac{1}{y}} \frac{|\widetilde{R}(\alpha)|^{2}}{|\alpha|} \mathrm{d} \alpha \\
& \ll N \log ^{2} N+\sum_{k=1}^{\mathcal{O}(\log y)} \frac{y}{2^{k}} \int_{\frac{2^{k}}{y}}^{\frac{2^{k+1}}{y}}|\widetilde{R}(\alpha)|^{2} \mathrm{~d} \alpha \\
& \ll N \log ^{2} N+\sum_{k=1}^{\mathcal{O}(\log y)} \frac{y}{2^{k}} N \frac{2^{k+1}}{y} \log ^{2} N \\
& \ll N \log ^{2} N \log y .
\end{aligned}
$$

End of the proof. Inserting (4.3) and (4.4)-(4.5) into (4.2) we immediately have

$$
\sum_{n=1}^{y} e^{-n / N} R(n)=\sum_{n=1}^{y} n e^{-n / N}+2 \sum_{n=1}^{y} e^{-n / N}(\psi(n)-n)+\mathcal{O}\left(N \log ^{2} N \log y\right) .
$$

Hence

$$
\sum_{n=1}^{y} e^{-n / N}[R(n)-(2 \psi(n)-n)] \ll N \log ^{2} N \log y,
$$

and the maximum of the right hand side is attained at $y=N$. Thus we can write

$$
\max _{y \in\left[N^{1 / 2}, N\right]}\left|\sum_{n=1}^{y}[R(n)-(2 \psi(n)-n)] e^{-n / N}\right| \ll N \log ^{3} N .
$$

Combining (4.1) and (4.6) we get that Theorem 1.2 is proved.

Remark. Let

$$
f(\alpha)=f_{N}(\alpha)= \begin{cases}\frac{1}{2} N^{1 / 2} \log N & \text { if }\|\alpha\| \leq(\log N)^{-1} \\ 0 & \text { if }\|\alpha\|>(\log N)^{-1} .\end{cases}
$$

Then $f$ satisfies both Lemma 2.1 and Lemma 2.3 in the sense that

$$
\int_{-\xi}^{\xi}|f(\alpha)|^{2} \mathrm{~d} \alpha \ll N \xi(\log N)^{2}
$$

for all $\xi \in[0,1 / 2]$, and

$$
\int_{-1 / 2}^{1 / 2}|f(\alpha)|^{2} \mathrm{~d} \alpha=\frac{1}{2} N \log N
$$

However,

$$
\begin{aligned}
\int_{1 / y}^{1 / 2} \frac{|f(\alpha)|^{2}}{\alpha} \mathrm{d} \alpha & =\frac{1}{4} N(\log N)^{2} \int_{1 / y}^{1 / \log N} \frac{\mathrm{d} \alpha}{\alpha} \\
& =\frac{1}{4} N(\log N)^{2} \log (y / \log N) \asymp N(\log N)^{3}
\end{aligned}
$$

for $y=N^{1 / 2}$ and sufficiently large $N$. This means that the crucial bound for $I_{3}(y)$ in (4.5) is essentially optimal in the present state of knowledge and that it cannot be 
improved without deeper information on $\widetilde{S}(\alpha)-z^{-1}$, such as the stronger analogue of Lemma 2.1 that follows from a suitable form of Montgomery's Pair-Correlation Conjecture.

\section{ACKNOWLEDGMENT}

The authors would like to thank Alberto Perelli for a discussion.

\section{REFERENCES}

[1] T. Apostol, Introduction to Analytic Number Theory, Springer-Verlag (1976). MR0434929 $(55: 7892)$

[2] G. Bhowmik, J.-C. Schlage-Puchta, Mean representation number of integers as the sum of primes, Nagoya Math. J., 200 (2010), 27-33. MR2747876

[3] H. Davenport, Multiplicative Number Theory, Springer-Verlag, 3rd ed. (2000). MR.1790423 (2001f:11001)

[4] A. Fujii, An additive problem of prime numbers, Acta Arith., 58 (1991), 173-179. MR.1121079 $(92 \mathrm{k}: 11106)$

[5] A. Fujii, An additive problem of prime numbers. II, Proc. Japan Acad. Ser. A Math. Sci., 67 (1991), 248-252. MR1137920 (93b:11132a)

[6] A. Fujii, An additive problem of prime numbers. III, Proc. Japan Acad. Ser. A Math. Sci., 67 (1991), 278-283. MR1137928 (93b:11132b)

[7] P. X. Gallagher, A large sieve density estimate near $\sigma=1$, Invent. Math., 11 (1970), 329-339. MR0279049 (43:4775)

[8] A. Granville, Refinements of Goldbach's conjecture, and the generalized Riemann hypothesis, Funct. Approx. Comment. Math., 37 (2007), 159-173. MR2357316 (2009b:11179)

[9] A. Granville, Corrigendum to "Refinements of Goldbach's conjecture, and the generalized Riemann hypothesis", Funct. Approx. Comment. Math., 38 (2008), 235-237. MR2492859 (2010c:11125)

[10] G. H. Hardy, J. E. Littlewood, Some problems of 'Partitio Numerorum'; III: On the expression of a number as a sum of primes, Acta Math., 44 (1923), 1-70. MR.1555183

[11] A. Languasco, Some refinements of error terms estimates for certain additive problems with primes, J. Number Theory, 81 (2000), 149-161. MR1743499 (2001h:11130)

[12] A. Languasco, A. Perelli, On Linnik's theorem on Goldbach number in short intervals and related problems, Ann. Inst. Fourier, 44 (1994), 307-322. MR1296733 (95g:11097)

[13] Y. Linnik, A new proof of the Goldbach-Vinogradow theorem, Rec. Math. N.S., 19 (1946), 3-8 (Russian). MR0018693 (8:317c)

[14] Y. Linnik, Some conditional theorems concerning the binary Goldbach problem, Izv. Akad. Nauk SSSR Ser. Mat., 16 (1952), 503-520 (Russian). MR0053961(14:847b)

Dipartimento di Matematica Pura e Applicata, Universitì di Padova, Via Trieste 63, 35121 Padova, ItALY

E-mail address: languasco@math.unipd.it

Dipartimento di Matematica, Università di Parma, Parco Area delle Scienze 53/A, Campus Universitario, 43124 Parma, Italy

E-mail address: alessandro.zaccagnini@unipr.it 